\title{
Traduire
}

\section{Écrire et traduire pour la scène : un même territoire du sensible}

Marianne Ségol et Karin Serres

\section{(2) OpenEdition}

Édition électronique

URL : http://journals.openedition.org/traduire/313

DOI : $10.4000 /$ traduire.313

ISSN : 2272-9992

Éditeur

Société française des traducteurs

\section{Édition imprimée}

Date de publication : 15 décembre 2010

Pagination : 102-113

ISSN : 0395-773X

\section{Référence électronique}

Marianne Ségol et Karin Serres, «Écrire et traduire pour la scène : un même territoire du sensible »,

Traduire [En ligne], 223 | 2010, mis en ligne le 10 février 2014, consulté le 22 septembre 2020. URL:

http://journals.openedition.org/traduire/313 ; DOI : https://doi.org/10.4000/traduire.313 


\section{Écrire et traduire pour la scène : un même territoire du sensible}

Marianne Ségol, Karin Serres

\section{LAB007 : vers un réseau de travail, d'expérimentation et de réflexion dans le théâtre contemporain européen pour la jeunesse}

Le théâtre dit " pour le jeune public " est en plein essor en Europe. Véritable art en direction de l'enfance et de la jeunesse, il se montre souvent aussi riche qu'original et inventif. Parce que cet engagement artistique diffère cependant selon les pays, en fonction de la place de l'enfant dans la société, de celle du théâtre dans l'art, de celle de l'écrit dans le théâtre, il nous semble enrichissant d'inventer aujourd'hui de nouvelles actions théâtrales transnationales pour croiser nos expériences, travailler et réfléchir ensemble pour faire avancer ce domaine profondément contemporain.

LAB007(1) vise à la circulation des écritures dramatiques contemporaines d'Europe pour l'enfance et la jeunesse ; à la rencontre active de leurs auteurs vivants, au partage de leurs expériences croisées ; à la mise en réseau des textes et des productions théâtrales pour l'enfance et la jeunesse qui en naîtront ; et à une réflexion commune et positive autour de toutes les énergies rassemblées dans ces processus de travail associés.

\section{Genèse du concept de traductions en duos}

Pour partager avec le jeune public la richesse du théâtre vivant qui s'écrit en Europe aujourd'hui, LABO07 encourage la traduction de pièces sélectionnées par ses comités de lecture et ce dans le plus grand nombre de langues possibles, le français constituant, selon les projets, la langue source ou la langue cible. LAB007 veut être un espace où toutes les formes de traduction théâtrale se pratiquent et s'inventent, dans la plus grande justesse par rapport au texte, à son projet de création et/ou d'édition.

(1) LAB007 a été fondé en 2007 par Karin Serres, Marianne Ségol, I'Institut Suédois, la SACD (Société des auteurs et compositeurs dramatiques), le Théâtre de la Tête Noire, le Théâtre de l'Est parisien et Massalia. 
Plus le traducteur travaille étroitement avec l'auteur de la pièce, plus le résultat est intéressant et juste. Seule cette collaboration étroite et cette compréhension profonde permettent la traduction du texte avec son contexte dont le traducteur devient le passeur. Et ce, d'autant plus qu'il s'agit d'un texte théâtral, chargé de tous ses non-dits, son sous-texte, ses codes théâtraux sous-jacents souvent très différents d'une culture à l'autre.

Outre la traduction classique en solo, Marianne Ségol (traductrice suédois/français) et Karin Serres (autrice dramatique) proposent à LAB007 de s'associer dans une expérience novatrice de traduction en duo, la grande richesse de ce duo résultant de la mise en commun de leurs deux pratiques de la langue théâtrale qu'elles devinent complémentaires. Elles proposent également de faire intervenir l'auteur de façon active à la phase finale de la traduction de sa pièce, lors d'une lecture bilingue en stéréo : le duo devient alors trio.

De nombreux organismes de traduction théâtrale européens s'intéressent à cette démarche de LAB007, comme Beaumarchais et la Maison Antoine Vitez en France, ou le Baltic Center en Suède, qui ont déjà soutenu certains de leurs projets.

\section{Première expérience de traduction en duo}

Pour leur première expérience de traduction en duo, Marianne Ségol et Karin Serres choisissent Brevet från NN (La lettre de NN) d'Erik Uddenberg, issue du premier choix de pièces suédoises jeune public envoyé par le comité de lecture suédois de LAB007.

Leur ambition est de mettre en pratique la traduction sous une forme plus vivante et expérimentale, en ayant en tête non seulement l'idée qu'un texte de théâtre doit être lu, mais aussi qu'il a pour vocation d'être joué sur une scène. L'idée d'un travail en duo entre un traducteur et un auteur n'est pas ici de combler un manque de compétence du traducteur mais plutôt de mettre en commun, tout au long du processus, leur expérience de la traduction et de l'écriture théâtrale afin de servir au mieux le texte. Ouvrir de nouvelles strates dans le texte, par échos de sens mais aussi par la création d'un territoire de non-dit et d'inexplicable.

\section{Le choix de la pièce}

La lettre de NN est une pièce qui s'inscrit dans la tradition suédoise d'écriture pour le jeune public en prise avec la réalité, où des thèmes sérieux sont traités avec pudeur et économie de mots. À travers une trame arbitraire, des scènes légères se mettent en place où le plus important n'est pas ce qui est dit, mais ce qui ne l'est pas. L'auteur semble épier chaque personnage depuis sa conduite de départ jusqu'à sa conduite d'arrivée. Dans cette écriture laconique, il raconte la richesse et la complexité des rapports humains. Cette pièce apparemment simple à traduire semble cacher bien des pièges. 


\section{Les différentes étapes de travail}

Elles travaillent d'abord chacune séparément : Marianne Ségol à partir du texte original, Karin Serres à partir de la traduction allemande (par Regine Elsässer) et ce, simplement pour, dans un premier jet personnel, trouver chacune leur vocabulaire de fond et les confronter. Ensuite, dès leur première séance de travail commune, elles écartent la traduction allemande pour ne plus se servir que de la version originale suédoise.

Et c'est mot par mot, ligne par ligne qu'elles passent en revue la pièce, avec l'idée de ne jamais gommer son étrangeté, les différences linguistiques et culturelles mais de les apprivoiser, de faire entendre ce que la langue exprime au-delà des mots, les "trous " d'interprétation, l'invisible profondément théâtral, la dimension scénique. Ce travail dans l'épaisseur, dans l'intimité du langage est ici plus qu'ailleurs une affaire de rythme, de musicalité... Progressivement, le travail devient de plus en plus intuitif : sentir le mot juste, les sonorités, le rythme, les silences, pour tenter de découvrir ce qui se cache derrière les mots, derrière les scènes apparemment anodines, pour saisir la vision particulière de l'auteur.

Elles démontent le texte suédois pour se l'approprier et en comprendre le sens visible et invisible. Elles le reconstruisent ensuite en pesant chaque mot, chaque expression. Avec en référence constante le ton, la voix de l'œuvre originale.

Dans un troisième temps, elles prennent contact avec l'auteur pour lui proposer une séance de travail autour de la traduction. Erik Uddenberg accepte l'invitation sans vraiment savoir ce qui l'attend. Et c'est à l'Institut Suédois, à Paris, que le trio se retrouve. Karin Serres et Marianne Ségol proposent alors une lecture en stéréo : Erik Uddenberg lira sa pièce en suédois en s'arrêtant après chaque scène, Karin Serres lira en écho la version française et Marianne Ségol écoutera les deux versions. L'effet que produit cette double lecture est une formidable écoute intuitive de la pièce, de son univers, de ses sonorités, de son souffle, de son ambiance générale. Si certains passages dans la version française accrochent, ils jaillissent aussitôt, comme par magie. II suffit alors de changer un ou deux mots, d'ajouter ou d'enlever une virgule, ensemble, en direct, pour que la langue retrouve sa fluidité. Dans cette ambiance très privilégiée, l'auteur se confie, donne des pistes, dévoile des parties cachées du texte, explique ce qui l'a poussé à écrire tel ou tel passage. Le duo de traduction entre dans l'ossature même de la pièce, dans son intimité la plus profonde. Un vrai moment de grâce !

Erik Uddenberg dira de cette séance de travail :

En participant à ce projet de traduction entre la Suède et la France, j'ai découvert un nouvel univers très stimulant. Moi qui ne connaissais du théâtre français que Molière et Koltès, j'ai découvert une société théâtrale très curieuse et large d'esprit, motivée par la recherche, et une tradition théâtrale centrée sur le texte. Pour moi, en tant qu'auteur, quel coup de fouet ! Ma plus belle expérience a été la façon de traduire à laquelle Karin Serres et Marianne Ségol m'ont 
initié pendant mon voyage à Paris. Après un travail préalable déjà très sensible, nous nous sommes assis ensemble. J'ai lu la première scène en suédois, Karin me l'a lue en français et Marianne, qui parle couramment les deux langues, écoutait. Et ainsi de suite. Entendre chaque ligne, chaque phrase, chaque mot de mon texte, de cette manière, en français et en suédois nous a permis à tous les trois de vérifier que le sous-texte de ma pièce Brevet från NN passait lui aussi entièrement d'une langue à l'autre. Cela doit devenir la dernière étape de toute traduction.

Pour Karin Serres et Marianne Ségol aussi, associer l'auteur au travail final de traduction s'est révélé être un moment si fort qu'il faudrait se donner le luxe de l'inscrire à chaque phase finale de traduction.

Afin de prolonger cette expérience novatrice si enrichissante et multiplier ses retombées tant théâtrales que linguistiques, elles lancent trois nouveaux types d'expériences dans le cadre de LAB007 : inverser le trio - ce sera l'aventure de Louise/les ours en Suède ; traduire de nouvelles pièces dans le cadre du duo : Inget växer utom Stig (och Molly) / Rien ne pousse sauf Stig (et Molly) et Dan då Dan dog / Le Mardi où Morty est Mort; et enfin, fortes de la découverte de l'extraordinaire richesse de cette collaboration, associer écriture et traduction dès les premiers mots d'un projet multilingue : le futur spectacle Rose, Rose, Rose.

\section{Inversion du trio : l'aventure de Louise/les ours en Suède}

La traduction d'un texte ouvrant un nouveau territoire commun entre l'auteur et ses traducteurs, Marianne Ségol et Karin Serres proposent de poursuivre le défrichage de ce territoire du sensible en inversant le sens de traduction : l'auteur traduit devient l'auteur associé à la traduction d'une pièce de l'auteur ex-traducteur. D'où, dès janvier 2008, la constitution d'un nouveau duo de traduction : Erik Uddenberg, l'auteur suédois de La lettre de NN, et Marie Kraft, traductrice du français vers le suédois, membre de LAB007, autour de la pièce Louise/les ours(2) de Karin Serres, qu'ils traduiront ensemble vers le suédois. Cette traduction en duo est soutenue par Beaumarchais-SACD.

Chaque duo doit créer sa propre méthodologie, trouver son équilibre, inventer son organisation, par tâtonnements, intuition et confiance mêlés. Marie Kraft et Erik Uddenberg, qui se connaissent déjà bien, trouvent comment travailler chacun de leur côté, à distance réelle (l'un étant en Suède, l'autre en France) mais avec des rendez-vous de travail réguliers dans un pays ou dans l'autre. II faudra souvent couper en suédois pour retrouver la poésie et le flot de

(2) Écrite pour partie au Canada (Banff playRite colony) en 2004, Louise/les ours est publiée à l'École des LoisirsThéâtre en 2006. Finaliste du Grand Prix de la littérature dramatique 2007, créée par P. Douchet en 2008, elle sera mise en scène par B. Algazi au Lavoir Moderne Parisien en 2010/2011. 
la langue de Karin Serres. L'écriture toute en retenue et évocations d'Erik Uddenberg sert du coup merveilleusement la langue sensuelle et poétique de l'autrice qui va à l'essentiel, qui capte la substance des relations des personnages entre eux et avec eux-mêmes. Fin août 2008, la traduction suédoise du nouveau duo est prête, sous le titre de Louise/Björnarna.

De septembre 2008 à juin 2009, Louise/les ours (version française et suédoise) circule avec trois autres pièces dans deux classes en Suède et deux classes en France via le projet "Comités de lecture jeunesse franco-suédois en milieu scolaire " mené par Pascale Grillandini (Postures) et Marianne Ségol, le Riksteatern (Théâtre National Itinérant suédois), I'Institut Suédois et LAB007. Louise/les ours sortira lauréate en Suède. Nul doute que la grande finesse de la traduction et sa sensibilité ont été pour beaucoup dans l'appréciation, par un public de jeunes suédois, de cette pièce.

Karin Serres dira de cette expérience :

Lors de la rencontre en Suède avec les deux classes, et des lectures théâtralisées, j'ai été très touchée de ne pas comprendre un mot de Louise/Björnarna mais d'y reconnaître la forme énergétique de Louise/les ours, son spectre sonore, le dessin de sa musique et l'enchaînement de ses émotions. Grâce à la traduction très fine d'Erik Uddenberg et Marie Kraft, ce passage dans un suédois si musical révélait une autre face de ma pièce, une autre vérité, continuité, un autre chemin possible pour la raconter.

Cette sélection par les jeunes et les discussions passionnantes qui l'accompagnent lors de la rencontre finale, en juin 2009, à Stockholm, incitent également le Riksteatern(3) à créer Louise/Björnarna à Stockholm le 8 mars 2011, dans une mise en scène de Driterö Kasapi. Quel aboutissement formidable et rapide, lorsqu'on sait que le théâtre suédois pour la jeunesse est déjà très riche d'écritures contemporaines qui se suffiraient à elles-mêmes.

\section{Même duo, nouvelles pièces}

Enthousiasmées par la découverte de leurs affinités mutuelles lors de leur traduction associée de La lettre de NN, et encouragées par LAB007, Marianne Ségol et Karin Serres souhaitent mettre leur duo à l'épreuve d'autres styles, d'autres langues théâtrales, travaillant toujours au passage vers le français de nouvelles pièces contemporaines pour le jeune public, envoyées par le comité suédois.

(3) http://www.riksteatern.se 
C'est d'abord Inget växer utom Stig (och Molly) / Rien ne pousse sauf Stig (et Molly) de Lucas Svensson(4), une pièce pour enfants, aussi comique et jouissive que La lettre de NN est sensible et poétique.

La langue actuelle et visuelle de Lucas Svensson facilite la projection imaginaire. Des thèmes comme la différence, la solitude, l'exclusion y sont développés mais sans aucune lourdeur. Les émotions vives et violentes sont exprimées par des répliques courtes, qui vont du tac au tac et dans une écriture fragmentée qui avance vite. Lucas Svensson refuse tout regard psychologique sur ses personnages, tout pathos. Au contraire, l'auteur met en avant l'humour de leur existence, de l'Existence, à partir de la métamorphose d'un jeune garçon, Stig, en monstre immense.

Comme pour La lettre de NN, chacune fait d'abord de son côté un premier jet de la pièce à partir d'une version différente : Marianne Ségol, à partir du texte original et Karin Serres, de la traduction allemande (par Jana Hallberg). L'idée étant de ne pas trop pousser les versions, de garder un texte brut, ouvert, très proche de la structure et de la syntaxe du texte original.

Dans un deuxième temps, plusieurs séances de travail s'organisent entre elles deux. La traduction allemande est mise de côté, la version originale suédoise étant bien sûr la version de référence.

Elles travaillent beaucoup l'humour de cette pièce décapante, sa rythmique particulière, la vivacité de l'échange de ses répliques. C'est un texte plein d'onomatopées qui engendre de longues discussions sur le ressenti intuitif des sons, des cris, dans chaque culture : une mine de différences passionnantes. Elles se concentrent aussi particulièrement sur les mots d'enfants prononcés en boucle par le héros, en litanies rassurantes, bien sûr différents dans leurs deux cultures, (kram gullis, pussipussi, puss stan... / câlin, poutou, bisou la ville...) et sur le patois scanien du père du héros, hilarant pour un public suédois capable de reconnaître l'accent spécifique de chaque ville, mais intraduisible dans un patois français, quel qu'il soit, car trop connoté. Raison pour laquelle elles optent finalement pour un langage oral plein d'élisions. Enfin, un traitement particulier est réservé aux didascalies, très poétiques, contrastant avec les comptines trash de Molly, entièrement à réinventer.

En juin 2008, le Théâtre de l'Est parisien accueille la lecture de la version française de cette pièce intitulée Rien ne pousse sauf Stig (et Molly), dirigée par Karin Serres, durant laquelle Marianne Ségol lit certains passages, en écho, en suédois, créant un monde encore plus irréel. La pièce est aussi envoyée à de nombreux comités de lecture et continue de circuler.

(4) Lucas Svensson, né en Suède en 1973, s'est formé au Dramatiska Institutet puis a travaillé dans de nombreux grands théâtres suédois. Depuis 2003, il est auteur associé au Dramaten (Théâtre royal) où il travaille également comme dramaturge. II a écrit une trentaine de pièces aussi bien pour les adultes que pour le jeune public. 
C'est ensuite avec Dan då Dan dog de Rasmus Lindberg(5), littéralement "Le jour (où) Dan mourut ", issu d'un nouvel envoi du comité suédois, qu'elles poursuivent, saison 2008-2009, leur travail de traduction en duo : des dialogues très contemporains, drastiques, pleins d'humour noir, de force et des scènes très visuelles bégaient et s'enchevêtrent dans une pièce pour adolescents où le normal et l'anormal se confondent, tout comme le rêve et la réalité s'entrechoquent avec une énergie et une humanité folles, le tout durant 24 heures pendant lesquelles, entre autres, un chien meurt.

À partir du même système de départ, (version suédoise et version anglaise, cette fois, de Gabriella Berggren), la traductrice et l'autrice associées rédigent chacune un premier jet autonome puis elles alternent séances de travail en commun et réécriture personnelle, le texte suédois redevenant très vite, comme toujours, la référence unique.

La langue de Rasmus Lindberg est aussi complexe que la construction et la temporalité de ses pièces. Dans une apparente multiplication de trajectoires physiques, d'histoires et de destinées, elle repose pourtant sur une construction abstraite, quasi mathématique, faisant appel à l'intuition quant à la rythmique de chaque passage et, surtout, quant à la façon dont les mots des uns font écho dans les textes des autres, bien qu'utilisés différemment, et dans d'autres registres de langage. Enfin, Rasmus Lindberg possède à merveille l'art de la répétition qui fait sens, un bégaiement aussi poétique qu'énergique, des redites qui ouvrent des brèches dans l'espace-temps de la pièce. Marianne Ségol et Karin Serres unissent donc toute leur expérience de traductrice et d'autrice, et partagent surtout leur intuition afin d'atteindre une version aussi élégante, légère et surprenante en français que la version suédoise et qu'elles intitulent, pour reprendre le jeu sonore du titre original : Le Mardi où Morty est Mort.

Cette pièce aussi originale que touchante est diffusée par LAB007 dans de nombreux comités de lecture où elle est très remarquée (Espace 600, Théâtre de la Tête Noire...), elle est également lue au Lundi en coulisses spécial LAB007 (Lyon, villa Gillet, juin 2010), est aussi choisie par le Répertoire Aneth et sera publiée en janvier 2011 aux éditions Espaces 34. Pour cette nouvelle pièce, quelle rapidité d'aboutissement. Et quel plaisir pour l'auteur d'apprendre qu'il sera bientôt publié en France, l'édition théâtrale étant si rare en Suède.

\section{Bilan de ces traductions en duo}

Soutenues par LAB007 et encouragées par les retours enthousiastes de nombreux professionnels de théâtre, Karin Serres et Marianne Ségol prévoient de nouvelles traductions théâtrales en duo, comme la nouvelle pièce de Rasmus Lindberg Den som lever får dö.

(5) Rasmus Lindberg, né en Suède en 1980, écrit aussi bien pour les adultes que pour le jeune public. II a fait sa percée avec Dan då Dan dog (Le Mardi où Morty est Mort), pièce sélectionnée par la Biennale de théâtre en 2007. II est aujourd'hui considéré comme l'un des jeunes auteurs suédois montants et s'inscrit dans une nouvelle génération d'auteurs à l'écriture très travaillée. 
Ces traductions en duo-trio qu'elles ont initiées pour LAB007 pourront plus tard se décliner avec d'autres auteurs dramatiques et traducteurs de théâtre, tout aussi passionnés qu'elles par le rapport langue-théâtre, réunis par affinités, goût de l'expérimental et qui mettront eux aussi leur professionnalisme au service de cette passation associée.

Toutes ces expériences additionnées de traductions inventives constituent peu à peu un terrain de fiction, de langue et de culture partagées qui crée une base artistique commune profonde, rare et partageable.

\section{écriture + traduction $=$ création}

Fortes de la découverte de l'interaction profonde qui existe entre leurs deux pratiques de la langue (celle de l'écriture et celle de la traduction) et touchées par leur enrichissement mutuel, Karin Serres et Marianne Ségol se lancent en juillet 2008 dans une toute nouvelle expérience de collaboration littéraire dramatique multilingue avec Malin Axelsson(6), autrice de théâtre suédoise. Pourquoi ce choix ? Par pures affinités instinctives entre les deux autrices qui se sentent mues par un même appétit d'écriture expérimentale et une même foi dans la richesse d'une rencontre dramaturgique, linguistique et théâtrale.

Si Malin Axelsson et Karin Serres écrivent chacune dans leur langue maternelle, elles font néanmoins le pari d'une écriture commune réelle grâce à la présence de Marianne Ségol, qui assumera une traduction immédiate et constante, dans les deux sens, du premier brouillon à la version finale, dans la plus grande confiance mutuelle (aucune des deux autrices n'a l'habitude de donner ses brouillons à lire). Toutes trois parient sur l'énergie créatrice déclenchée par une telle circulation linguistique. Elles constituent donc un trio d'écriture atypique - deux autrices, une traductrice - qui travaille en trois langues : le suédois, le français et l'anglais, langue véhiculaire.

Lors d'un premier atelier d'une semaine, à Paris, en juin 2008, elles commencent de façon très subjective par échanger des textes (documentaires, analytiques, fictionnels), des images, des réflexions, de la musique, issus de leurs différentes cultures ou générations et liés à la question " fille/garçon ". Mis en commun selon une structure puzzlistique, tous ces éléments bâtissent progressivement leur territoire commun à partir duquel elles vont écrire ce spectacle multilingue destiné aux enfants, aux adolescents et aux adultes, tournant autour de la vertigineuse question des genres.

(6) Née en 1975 en Suède, Malin Axelsson est autrice, dramaturge, metteuse en scène et, depuis 2009, directrice du théâtre Ung Scen/Öst de Linköping (Suède), célèbre scène jeune public. Ses pièces d'avant-garde ont fait le tour de la Suède et plusieurs ont été traduites. 
De retour chacune chez elles, Malin Axelsson et Karin Serres écrivent leurs premiers textes de fiction, monologues, dialogues, chansons, qu'elles s'échangent par mail, via Marianne Ségol qui les traduit, en l'état, constituant petit à petit un double corpus de brouillons bilingues, rigoureusement symétrique.

Après ces échanges virtuels, le trio d'écriture/traduction se retrouve en mai 2009 pour un premier workshop au Théâtre de l'Est parisien (Paris, France), avec trois comédiens français de la troupe du théâtre. Ce workshop, qui alterne discussions, lectures et improvisations, est mené en français et en anglais par le trio. S'y ajoute une initiation au suédois portée par Marianne Ségol et Malin Axelsson qui ouvre de nouveaux espaces de fiction issus de l'étrangeté à dire et entendre une langue inconnue sur scène.

Ce premier temps de travail du texte révèle un glissement thématique du projet vers la notion du " Devenir une fille/devenir un garçon ", avec un fort ancrage dans l'adolescence. À l'issue de ce workshop, les comédiens et les trois autrices donnent une lecture multilingue publique d'une partie du corpus, travaillée en français mais ponctuée d'incursions en suédois et en anglais et suivie d'une discussion avec le public. La présence des langues étrangères sur une scène révèle leur surprenante puissance dramaturgique.

Stimulées par ce retour positif, les deux autrices repartent retravailler leurs textes initiaux et en écrire de nouveaux que la traductrice continue à traduire dans les deux sens. Le fait d'être présente dès les premiers balbutiements d'un texte, d'en traduire plusieurs versions successives, de plus en plus mûres, de plus en plus précises, permet à la traductrice un apprivoisement de la situation et des personnages en train de naître dans la fiction, de la langue de chaque autrice et du registre linguistique global du projet.

En janvier 2010, un second workshop se déroule en Suède, au théâtre Ung Scen/Öst de Linköping, dont Malin Axelsson vient d'être nommée directrice. Menée cette fois en suédois et anglais par les deux autrices et la traductrice, avec deux comédiens suédois de la troupe du théâtre, cette nouvelle semaine de travail révèle la même puissance de la langue étrangère non parlée mais jouée sur scène : cette fois-ci le français. Elles commencent à constituer un lexique trilingue dans lequel chacune pourra puiser. Sonorités, expressions, associations d'idées, la fiction rebondit entre les trois langues et ce ricochet est lui-même générateur de fiction. Trois langues, car à la grande surprise du trio, l'anglais glisse de langue véhiculaire à langue de fiction. Dans certains textes, l'anglais sonne plus juste, permet une liberté plus grande. Ce travail révèle et souligne ce que la langue dévoile de la culture d'un pays. On aborde là la richesse de ce projet dont le trilinguisme multiplie les possibilités théâtrales. Cette nouvelle étape entraîne la fiction en cours d'écriture encore plus largement vers "la question du genre " et "les métamorphoses du corps".

En avril 2010, le trio mène un troisième workshop au Théâtre de la Tête Noire (Saran, France), en français et en anglais, pendant le festival "Text'avril " consacré à l'écriture contemporaine, 
dont le thème est "L'intime ". Toujours mû par une même soif d'expérimenter d'autres façons de travailler, le trio y associe une nouvelle expérience de collectage auprès de groupes de gens d'âges et de milieux très différents, à partir d'un questionnaire personnel et sensoriel élaboré en commun qu'elles tenteront ensuite d'intégrer dans la fiction. Suit une brève étape de réécriture afin de composer un nouveau corpus. Le trio travaille ensuite avec cinq comédiens de la troupe du festival pour aboutir à une nouvelle lecture multilingue de cette étape de travail suivie, comme à chaque fois, d'une discussion avec le public.

Les comédiens parlant presque tous des langues étrangères, un véritable engouement naît chez eux pour ce jeu de langues que le trio travaille, générant un apport ponctuel de nouveaux mots dans de nouvelles langues : espagnols, vietnamiens, lituaniens, anglais. II ne s'agit pas de se transformer en tour de Babel théâtrale, mais bien de travailler la spécificité du rapport langues/scène. La " Babel-langue " naît lorsqu'elle est justifiée d'un point de vue dramaturgique.

Chaque nouvelle occasion permettant d'approfondir leur recherche artistique, littéraire, linguistique et théâtrale, le trio réalise maintenant que le cœur de ce futur spectacle est « l'identité " : ce qui fait que nous sommes nous-mêmes, au milieu de la foule, dans la société.

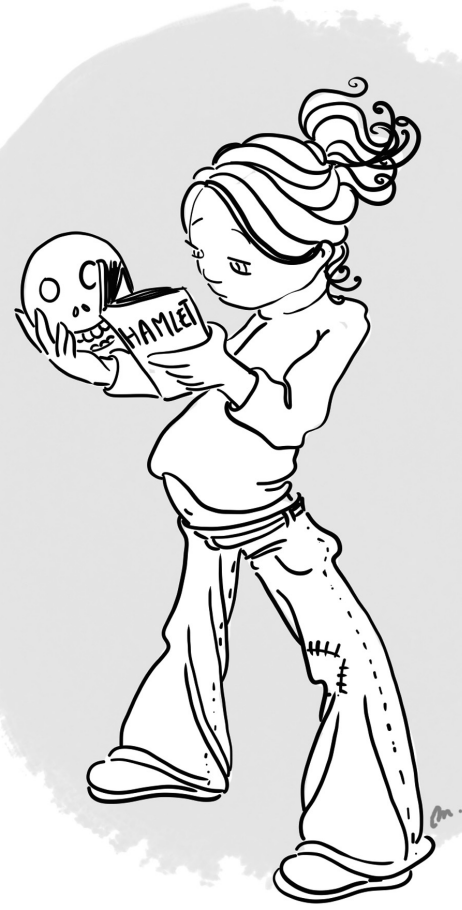

Illustration : Marlène Junius, http://alotoftralala.over-blog.com 
Enfin, en juillet 2010, un nouveau workshop en France réunit Marianne Ségol et Karin Serres au Conservatoire d'Avignon, dans le cadre du festival, à l'invitation du directeur qui souhaite initier l'ensemble de ses élèves au théâtre jeune public européen. Ces jeunes comédiens volontaires ont en commun un grand appétit pour les langues. Leurs esprits ouverts prennent un grand plaisir à expérimenter et se découvrent par exemple une grande aisance à jouer tout un texte en suédois sans en parler un mot, créant ainsi une nouvelle langue scénique.

Grâce à cette équipe, Marianne Ségol et Karin Serres peuvent prolonger ce travail passionnant sur la langue étrangère en tant que langue habitable, " incarnable ", même et surtout si on ne la parle pas. Les comédiens expriment leur grand plaisir éprouvé, la libération du corps et de l'expression que cette étrange interprétation de textes français truffés d'échos en langues étrangères leur procure : " On dit mieux parce qu'on reprend, la langue étrangère semble appuyer le propos, donc renforce notre interprétation, c'est un support pour nous, une force accrue. "

Saison 2010-2011, il reste maintenant une année au trio pour finir d'écrire Rose, Rose, Rose, cette fiction pour tout public multilingue centrée sur le rapport corps-identité.

Leur désir est de continuer à travailler la force dramaturgique de toutes ces langues, ce qu'elles permettent d'exprimer, par quel moyen elles y arrivent et tous les échos possibles entre elles.

À l'aube de leur troisième et dernière année d'expérimentation partagée, juste avant la création du spectacle à l'automne 2011, un nouveau rendez-vous se profile : une présentation publique, lors du congrès mondial Assitej, dans cette richesse linguistique et culturelle vers laquelle le projet se développe.

Tous les partenaires de ce projet suivent attentivement son déroulement, et plusieurs lieux se sont d'ores et déjà inscrits sur sa production, dont le théâtre Ung Scen/Öst de Linköping où il sera créé, dans une co-mise en scène de K. Serres et M. Axelsson.

D'un désir d'écriture en commun, deux autrices se sont associées avec une traductrice afin de constituer dès leurs premiers mots un territoire linguistique partagé, tant sur le plan du texte que du contexte. Le fait d'intégrer la traductrice dès le début du processus d'écriture a profondément enrichi la fiction, permettant une circulation aussi inédite que rapide de l'une à l'autre, et révélant ce que la langue véhicule de sa propre culture : la langue en tant que reflet d'une identité qui se construit en permanence.

Depuis leur projet d'écrire en deux langues (le français et le suédois), leur territoire linguistique ne cesse de se déployer. Car la force du rapport entre les langues (maternelle, étrangère, incompréhensible, supposable...) et la scène fait considérablement avancer la fiction qui renvoie les trois autrices sur le territoire de l'identité : notre langue nous définit.

Et la langue étrangère devient une langue du sensible, du non-dit que les interprètes comme le public doivent aborder différemment, plus intuitivement. 
Si la langue est au cœur du théâtre, expression humaine si complexe et subtile, la rencontre entre plusieurs langues sur scène ouvre sur d'encore plus grandes richesses et possibilités, exacerbées par les échos entre elles et le terrain du non-dit, de l'invisible si porteur de sens qu'ils créent. Enracinées dans le sensible de la langue théâtrale, traduction et écriture sont si profondément liées que leur association se révèle créatrice, toutes deux forces vives des dramaturgies contemporaines.

Ce qui importe, c'est de travailler d'une façon inventive dans le vivant du théâtre européen, pour et avec le public d'aujourd'hui, dans la plus grande ouverture possible. La langue dans tous ses sens semble pouvoir en être à la fois le cœur, le corps, le sang, l'énergie et le mouvement.

karin@karinserres.com mariannesegol@hotmail.com

septembre 2010

Marianne SÉGOL, née en 1972, est traductrice de pièces de théâtre et de littérature suédoises vers le français. Passionnée par l'écriture dramatique contemporaine pour le jeune public, elle se rend régulièrement en Suède pour découvrir des créations, rencontrer des auteurs, des directeurs de théâtre et des agents, la Suède ayant une longue et intéressante tradition d'écriture pour la jeunesse. Membre fondatrice de LAB007, elle fait aussi partie du comité nordique de la Maison Antoine Vitez. Elle a traduit des auteurs de théâtre comme Lucas Svensson, Staffan Göthe, Suzanne Osten, Rasmus Lindberg, Sara Stridsberg... et des auteurs de romans comme Astrid Lindgren, Håkan Nesser, Henning Mankell, Mats Wahl, Moni-Nilsson Brännström...

Karin SERRES, née en 1967, est autrice, metteuse en scène, décoratrice et traductrice de théâtre. Elle a écrit une cinquantaine de pièces dont plus de la moitié pour jeune public/tout public. Une vingtaine de ses pièces a été créée, certaines par elle-même, et une quinzaine éditée. Elle écrit aussi des pièces radiophoniques, des romans, des chansons, des albums et des feuilletons. Elle aime travailler en lien avec la vie de différents lieux, en France comme à l'étranger, et ses pièces sont traduites en anglais, allemand, suédois et portugais. Elle traduit aussi des pièces d'autres auteurs, de l'allemand ou de l'anglais vers le français. Après les Coq Cig Gru, collectif agitateur d'écriture théâtrale pour tout public, elle est membre fondatrice de LABOOT.

- Plus d'informations sur LAB007 et/ou sur ces expériences : www.laboo7.eu

- Première anglaise du feuilleton théâtral multilingue "Ank! Ang! je suis un Oi sauvage" lors du festival Theatre Cafe / Company of Angels à Londres (Royaume Uni), le 6 novembre 2010.

- Prochain rendez-vous Rose, Rose, Rose:

Première présentation multilingue au 17e Congrès Mondial Assitej, Copenhague, Malmö (Danemark, Suède), mai 2011. 